\title{
A Visualization Based Approach for Digital Signature Authentication
}

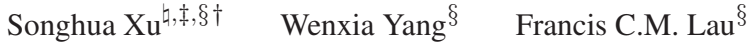 \\ ${ }^{\natural}$ College of Computer Science and Technology, Zhejiang University, Hangzhou, Zhejiang, P.R. China, 310027 \\ $\ddagger$ Department of Computer Science, Yale University, New Haven, Connecticut, USA, 06520 \\ $\S$ Department of Computer Science, The University of Hong Kong, Hong Kong, P.R. China
}

\begin{abstract}
We propose a visualization based approach for digital signature authentication. Using our method, the speed and pressure aspects of a digital signature process can be clearly and intuitively conveyed to the user for digital signature authentication. Our design takes into account both the expressiveness and aesthetics of the derived visual patterns. With the visual aid provided by our method, digital signatures can be authenticated with better accuracy than using existing methods-even novices can examine the authenticity of a digital signature in most situations using our method. To validate the effectiveness of our method, we conducted a comprehensive user study which confirms positively the advantages of our approach. Our method can be employed as a new security enhancement measure for a range of business and legal applications in reality which involve digital signature authorization and authentication.

Categories and Subject Descriptors (according to ACM CCS): I.3.3 [Computer Graphics]: Picture/Image Generation_-Viewing algorithms; I.3.m [Computer Graphics]: Miscellaneous_Visualization; H.1.2 [Information Systems]: User/Machine Systems-Human factors, Human information processing
\end{abstract}

\section{Introduction}

We are in a digital era where there is an increasing trend to digitize any object in reality. Human signature is one such object. It is now common in many supermarkets where credit card payment can be digitally signed using a tablet pen. Digital signatures however, like conventional signatures, can be unsafe; once a digital signature is revealed to the public, it is subject to being forged. For example, the signatures of Fig. 7(I- $\left.O_{1}\right)$ and Fig. 7(I- $\left.F_{1}\right)$ are extremely similar but the latter is in fact an imitation of the former. Nevertheless, digital signatures have added features which are not present in conventional signatures. These features include the speed and pressure of the signing when the signature was recorded. These additional information can be used to strengthen the signature authentication procedure, which motivates this paper.

$\dagger$ Contact him at songhua DOT xu AT yale DOT edu.
Over the past several decades, researchers have proposed many automatic signature authentication and writer verification algorithms. Despite the efforts, these intelligent algorithms still tend to fail to perform satisfactorily for the complicated cases. Deviating from the approaches taken by these previous efforts, in this paper, we pursue a visualizationbased approach to digital signature authentication. The study reported in [BNPH02] suggests that one's writing habit can be largely considered a distinctive mark of the person; and statistically, the writing speed and pressure of a person's digital handwriting are sufficient to uniquely represent the person's writing habit. In this paper, we propose a visualization metaphor for illustrating personal handwriting pattern in a digital signature process. The notable feature of our method is its ability to visually convey the speed and pressure of the digital handwriting process. Our metaphor for visualizing digital signature presents an easy-to-examine visual pattern to enable even the untrained eyes to conveniently authenticate digital signatures with a high degree of accuracy. Our approach should be able to improve the accuracy and relia- 
bility of digital signature authentication as it is significantly more challenging to forge a signature matching not just the shape of a signature but also the writing habit of a person.

The rest of our paper is organized as follows. We first survey some closely related work in Sec. 2. Next, we explain the design of our signature visualization method in Sec. 3, which is followed by some selected experiment results in Sec. 4. After that, we report a comprehensive user study in Sec. 5, which validates our approach. Finally we conclude the work in Sec. 6 .

\section{Related Work}

We first look at some most related work to our studies here. Bensefia et al. [BNPH02] introduced the concept of inter-writer invariant which is detected using an automatic classification procedure. Schomaker and Bulacu suggested using connected components and edge-based features for writer identification and verification [SB04]. Bensefia et al. [BPH05] suggested the use of graphemes extracted from segmentation of cursive handwritings and other local features. Schlapbach and Bunke [SB07] employed HMM based recognizers. Bulacu and Schomaker [BS07] proposed a text independent writer identification and verification technique based on textural and allographic features. Also related to our study is prior studies on using pen dynamic information for writer verification. Kikuchi and Akamatsu [KA01] studied a hardware-based approach for writer identification based on writing pressure. Schimke et al. [SVD04] proposed an online signature authentication method based on an eventstring model to represent the features derived from the signature process, and they used Levenshtien distance for comparing signatures.

\section{Our Visualization Method}

In this paper, we rely on the statistical distribution of three features-writing speed, writing direction and pen pressure-during digital signature to visualize the writing habit of a person. The challenge of our visualization design is that we need to present the distribution of the three features in a single graph such that it is easy to read and compare. Traditional statistical graphs, e.g., histogram, pie chart and statistical curve, are not suitable choices because they are not designed for visualizing multi-features in a single graph. We introduce the idea of a "digital lily" (as shown in Fig. 1(a)), which consists of a petal, several pistils and an anther, to represent the digital signature habit of a person. The merits of a digital lily include not only its attractive appearance but also its expressiveness - we can use the shape and texture of the petal, pistils and the anther to convey the key characteristics of a person's digital handwriting process.

In the remainder of this section, we will first look at how to collect and pre-process data from a digital signature process. After that, we examine our design of using the digital lily to visually reveal the characteristic information on the writing speed and pressure aspects of a handwriting process.

\subsection{Data Collection from Digital Handwriting Processes}

We sample two types of information in a digital handwriting process: the writing speed and pen pressure as expressed by a user during his handwriting process. The acquired signals come in a data stream. Assume $t_{0}$ is the starting moment of a digital signature process. We periodically read signals from our digital pen in the form of a triplet $\left(x_{t}, y_{t}, p_{t}\right)$ where $\left(x_{t}, y_{t}\right)$ specifies the location of the pen tip at time $t$, and $p_{t}$ is the pen tip pressure at the time. We normalize the sampled pressure values to the range of $[0,1]$. From two adjacent time moments and their pen tip locations, we can derive the velocity of the pen $v_{t}=\left(v_{x, t}, v_{y, t}\right)$ through finite difference, i.e., $v_{x, t}=\frac{x_{t+\Delta t}-x_{t}-\Delta t}{2 \Delta t}$ and $v_{y, t}=\frac{y_{t+\Delta t}-y_{t}-\Delta t}{2 \Delta t}$ where $\Delta t$ is our device's signal sampling period. Given $v_{t}$, we can calculate its magnitude, $\left|v_{t}\right|=\sqrt{v_{x, t}^{2}+v_{y, t}^{2}}$, and its direction, $\operatorname{dir}\left(v_{t}\right)=\operatorname{atan} 2\left(v_{x, t}, v_{y, t}\right)$ where $\operatorname{atan} 2(x, y)$ computes the angle in radians between the positive segment of the $\mathrm{X}$ axis and the point given by the coordinates $(x, y)$ on the plane. atan $2(x, y)$ 's range is between $[0,2 \pi)$ (see http://en.wikipedia.org/wiki/Atan2 for more explanations on this function definition). The derived triplets in the form of $\left(\left|v_{t}\right|, \operatorname{dir}\left(v_{t}\right), p_{t}\right)$ are used in our visualization.

\subsection{Digital Lily Design for Handwriting Visualization}

An example digital lily is shown in Fig. 1(a) where we use the petal and pistils of the lily to show the statistical distributions of the writing speed and pressure respectively. The anther of a digital lily, which is mounted on the end of the longest pistil, shows the distribution of writing direction during the digital signature process. We use these elements of our digital lily to visualize a person's writing habit for signature authentication.

\subsubsection{Petal of a Digital Lily}

A digital lily in our design has one petal (see Fig. 1(a)). We use the texture of the petal to represent the range within which the magnitude of the writing speed (or just magnitude from now on) of all the writing samples in a digital signature process would vary, and we use the contour of the petal to visualize the statistical distribution of the magnitude. A petal has the following visual properties:

Contour of the petal Fig. 1(b) illustrates the procedure for deriving the contour of a petal. First, we set up a Cartesian coordinate system, whose origin $O$ is the center of the displaying window. Second, we equally divide the range of the magnitude of all the writing samples $\left[0, v_{\max }\right]$ into $\gamma$ subranges, $Q_{1}, \cdots, Q_{\gamma}$, where $Q_{i}=\left[(i-1) \cdot v_{\max } / \gamma, i\right.$. $\left.v_{\max } / \gamma\right) \quad(i=1, \cdots, \gamma-1)$ and $Q_{i}=\left[(i-1) \cdot v_{\max } / \gamma, i\right.$. 


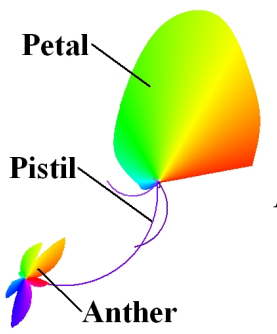

(a)

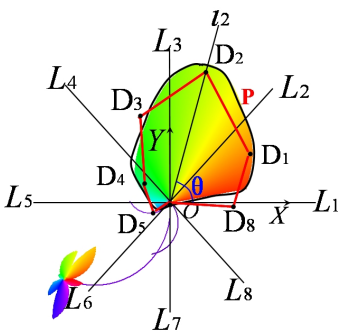

(b)
Figure 1: The composition of a digital lily. (a) shows the digital lily derived from the handwriting in Fig. 7(I-O $\left.O_{1}\right)$. (b) illustrates the procedure to determine the contour of the petal in (a).

$\left.v_{\max } / \gamma\right] \quad(i=\gamma)$. For simplicity and ease of reading, in Fig.1(b), we set $\gamma$ to be 8 . In all our actual experiments, we set $\gamma$ to be 18 . For practical deployment, users can optimally tune the $\gamma$ value to match different displaying devices. We also equally split the entire screen space into $\gamma$ regions using $\gamma$ radical lines, $\overrightarrow{O L_{1}}, \cdots, \overrightarrow{O L_{\gamma}}$ as shown in Fig. 1(b). The region spanned by the radical lines $\overrightarrow{O L_{i}}$ and $\overrightarrow{O L_{i+1}}$ corresponds to the subrange $Q_{i}$ of writing samples as derived earlier. Thirdly, we count the number of writing samples whose handwriting speed's magnitude is within $Q_{i}$. We denote this number as $l_{i}$. We also calculate the mean magnitude of the writing samples belonging to the region $Q_{i}$ as $|v|_{i}^{\text {mean }}$. Fourthly, we map $|v|_{i}^{\text {mean }}$ into an angle according to $\theta\left(|v|_{i}^{\text {mean }}\right)=\left\lfloor 360 \cdot \frac{|v|_{i}^{\text {mean }}}{v_{\text {max }}}\right\rfloor$. This linear mapping transforms the magnitude $|v|_{i}^{\text {mean }}$ from the range of $\left[0, v_{\max }\right]$ into the range of $[0,360]$. Next, we draw a radical line $i_{i}$, which spans an angle of $\theta\left(|v|_{i}^{\text {mean }}\right)$ with the positive segment of the $X$ axis. After that, we find a point $D_{i}$ on $\mathrm{\imath}_{i}$, where $\left|\overline{O D_{i}}\right|=\kappa_{\text {petal }} \cdot \frac{l_{i}}{\sum_{i=1}^{\gamma} l_{i}}$, in which $\kappa_{\text {petal }}$ is a tunable parameter adaptive to the size of the displaying window. Here we use $O D_{i}$ to represent the statistical distribution of the magnitude of writing speed samples in the region $Q_{i}$. Note that for each region $Q_{i}$, we can thus obtain an end point position $D_{i}$. Since we have $\gamma$ regions in a petal, this leads to $\gamma$ such position points, one for each magnitude region $Q_{i}$. All these points form an ordered discrete curve $P=\overline{D_{1}, D_{2}, \cdots, D_{\gamma}, D_{1}}$. Finally, we fit a degree-3 piecewise Bezier curve in the least squares optimal sense to get a smooth curve representation for $P$ (see Fig. 1(b)). The resultant Bezier curve forms the contour of the petal.

Texture inside a petal We derive the texture inside a petal as follows. The brightness of the color is always set to 0.5. The changing components are the hue and the saturation. For an arbitrary point $P_{t}$ inside the petal whose polar angle is $\theta_{t}$, its hue value is also set as $\theta_{t}$. To determine the saturation value for $P_{t}$, we first draw a line $\overline{O P_{t}}$ (see Fig. 2(b)). Assuming that this line or the extension of

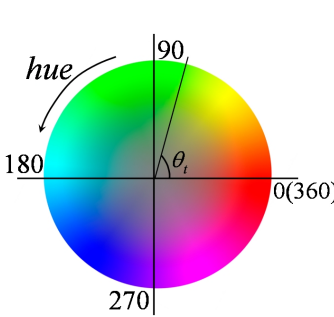

(a)

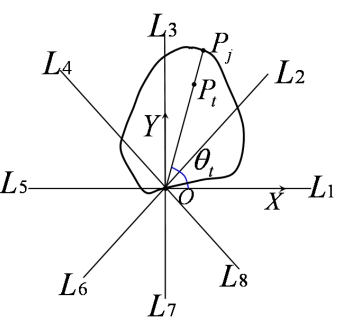

(b)
Figure 2: Deriving the texture for a petal in a digital lily. (a) gives a standard HSB color circle. (b) shows how to deriving the texture for the petal in Fig. 1.(a) by mapping the texture of the circle shown in (a) to the petal shape.

this line intersects with the contour of the petal at point $P_{j}$, then $P_{t}$ 's saturation is set as the relative distance of $P_{t}$ to the origin of the coordinate system $O$ with respect to $P_{j}$ 's distance to the origin, i.e., $\left|\overline{O P_{t}}\right| /\left|\overline{O P_{j}}\right|$. This amounts to distorting the standard HSB color circle (see Fig. 2(a)) to fit the shape of the petal (see Fig. 2(b)). The resultant texture mapped petal can be seen in Fig. 1(a).

\subsubsection{Pistils of a Digital Lily}

Similar to the case of a petal visualizing the distribution of handwriting magnitude for a group of writing samples as explained in Sec. 3.2.1, we use each pistil of a digital lily to visualize the distribution of handwriting pressure for a group of writing samples. Analogous to the generation of a petal, we first uniformly divide the range of all the possible pressure values into $\beta$ equal sized regions, $R_{1}, R_{2}, \cdots, R_{\beta}$. We also equally divide the whole displaying region into $\beta$ parts using $\beta$ radical lines, $\overrightarrow{O L_{1}}, \overrightarrow{O L_{2}}, \cdots, \overrightarrow{O L_{\beta}}$ (see Fig. 3). Here $\beta$ is a user tunable parameter controlling the complexity of the visualization pattern. Users can tune the value to best fit their displaying devices. In all the experiments reported in this paper, $\beta$ is set to 6 . We denote the number of writing samples falling into the region of $R_{i}$ as $m_{i}$. A pistil has the following visual properties:

Shape of a pistil The shape of a pistil is an arc, which has a constant span angle. In this paper, the angle is fixed as 120 degrees all the time (see Fig. 3). To determine

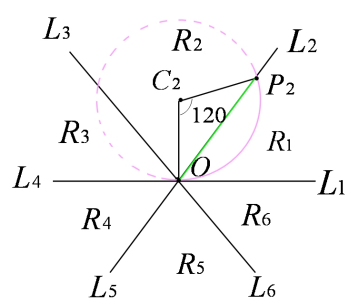

Figure 3: Determining the shape of a pistil. 
the shape for the $i$-th pistil, we first draw a line segment $\overline{O P_{i}}$, whose direction aligns with the boundary line $\overrightarrow{O L_{i}}$. The length of $\overline{O P_{i}}$ is determined according to the percentage of writing samples falling into the region of $R_{i}$, i.e., $\left|\overline{O P_{i}}\right|=\kappa_{\text {pistil }} \cdot m_{i} / \sum_{j=1}^{\beta} m_{j}$ where $\kappa_{\text {pistil }}$ is user tunable whose default value is set as half of the height or width of the resultant digital lily image, whichever is smaller. Given the line segment $\overline{O P}$, we can draw a circle $C_{i}$ whose circumference passes through both end points of $\overline{O P_{i}}$ and whose central angle spanned by $\overline{O P_{i}}$ is 120 degrees (see Fig. 3). In general, the minor arc of $\widehat{O P}_{i}$ forms the shape of the $i$-th pistil.

Color of a pistil Every pistil has a uniform color. The hue of every pistil is set to purple, i.e., 270.0; its saturation is always set to 1.0 . The only changing component in the color composition is its brightness term, which indicates the average pressure of the writing samples represented by a pistil - the higher the average pressure, the darker the color of the pistil. Assuming that the average pressure of the writing samples in the pistil is $\overline{p r e}$ and the maximum of all the writing samples acquired throughout the entire handwriting process is pre $_{\max }$, then the brightness of the pistil is set to $\overline{\text { pre }} /$ premax.

\subsubsection{Anther of a Digital Lily}

A digital lily has one anther, which consists of multiple flakes. We use the anther to visualize the statistical distribution of the writing directions during a signature process. To do this, we first equally divide the angle range of all the possible handwriting directions $[0,360)$ into $\alpha$ sub-ranges, denoted as $S_{1}, S_{2}, \cdots, S_{\alpha}$. And then we classify all the writing samples of a signature process into $\alpha$ groups according to their writing directions. That is, a given writing example $v_{t}$ will be assigned to a range $S_{i}$ according to its writing direction $\operatorname{dir}\left(v_{t}\right)$, where $i=\left\lfloor\operatorname{dir}\left(v_{t}\right) \cdot \alpha /(2 \pi)\right\rfloor$. Each $S_{i}$ is represented by an anther flake. More concretely, we denote the number of writing samples falling into the range of $S_{i}$ as $n_{i}$. Here $\alpha$ is a user tunable variable depending on the resolution of the displaying devices. In all the experiments reported in this paper, $\alpha$ is always set to 10 . The anther and its flakes have the following visual properties:

Location of the anther The anther of a digital lily always lies at the end of the longest pistil of the digital lily. Such a design decision is purposely made to draw the viewer's attention to the longest pistil. Recall that the longest pistil corresponds to the group of writing samples whose handwriting directions are predominant. In case two pistils have the same length, which in practice would almost never happen, we randomly select one of them to grow the anther.

Orientation of an anther flake The orientation of the $i$-th anther flake, $\phi_{i}$, is aligned with the mean handwriting direction of the writing samples represented by the anther flake. Recall that each anther flake represents all the writing samples belonging to a certain $S_{i}$.

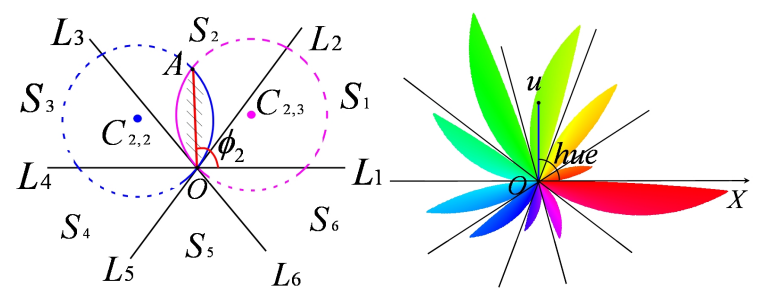

(a)

(b)

Figure 4: Determining the shape of an anther flake (a) and its interior texture (b).

Contour of an anther flake The contour of an anther flake consists of two arcs. The process of how to derive the contour of an anther flake is explained below, and illustrated in Fig. 4(a). First we equally divide the whole plane into $\alpha$ sectors $S_{1}, \cdots, S_{\alpha}$ using $\alpha$ boundary lines $L_{1}, L_{2}, \cdots, L_{\alpha}$. For simplicity and ease of reading, in Fig. 4(a) we show the situation when $\alpha$ is 6 . Each sector $S_{i}$ allocates the space for drawing an anther flake. Without loss of generality, we look at how to draw an anther flake in the sector of $S_{2}$ below. Accordingly, in our following discussion, the subscript $i$ always equals to 2 . Given the orientation $\phi_{i}$ of the $i$-th anther flake as determined by the procedure above, we first draw a line segment $\overline{O A}$ following the direction of $\phi_{i}$. The length of the line segment is proportional to the number of writing samples that the anther flake represents, i.e., $n_{i}$. In case $n_{i}=0$, the anther flake will disappear and nothing will be drawn. Once the line segment $\overline{O A}$ has been plotted, we can draw two circles $C_{i, i}$ and $C_{i, i+1}$. In our context, these two circles are $C_{2,2}$ and $C_{2,3}$. The circle of $C_{i, i+1}\left(C_{2,3}\right)$ has its circumference passing through the two end points of $\overline{O A}$, which is also tangent to the line $L_{i+1}\left(L_{3}\right)$. Similarly, the circle of $C_{i, i}\left(C_{2,2}\right)$ has its circumference passing through the two end points of $\overline{O A}$ and tangent to the line $L_{i}\left(L_{2}\right)$. The overlapping area between $C_{i, i}$ and $C_{i, i+1}$, i.e. $C_{2,2}$ and $C_{2,3}$, forms the interior area of the second anther flake.

Texture of an anther flake The texture inside an anther flake is used to indicate the distribution of handwriting directions of the writing samples that an anther flake represents. For any place $u$ inside the anther flake, its color is determined as follows. We fix the color's brightness and saturation components to be 0.5 and 1.0 respectively; the color's hue value is taken as the angle spanned by the line segment $\overline{\mathrm{Ou}}$ and the positive segment of the $X$ axis (see Fig. 4(b)).

\section{Experimental Results}

We implemented and tested our visualization method on a desktop PC equipped with 2 GB memory, an Intel Core 2 Duo CPU E6750 @ 2.66GHz, and a Wacom PTZ-431W tablet. For all the experimental results reported here, realtime response has been achieved. 

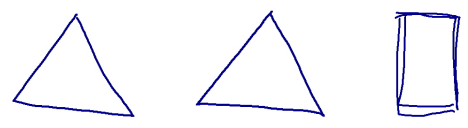

(I-original)
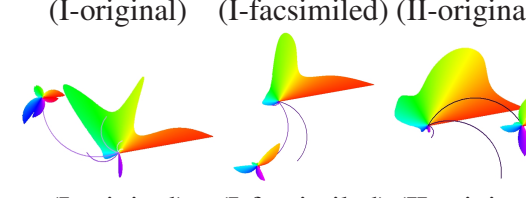

(I-original)

(I-facsimiled)
(II-original) (II-facsimiled)

Figure 5: Two pairs of simple handwriting pieces by two persons, where the second person tries to facsimile the writing of the first one. The first row shows their handwritings, which look very similar; the second row shows their corresponding digital lilies, which appear very different.

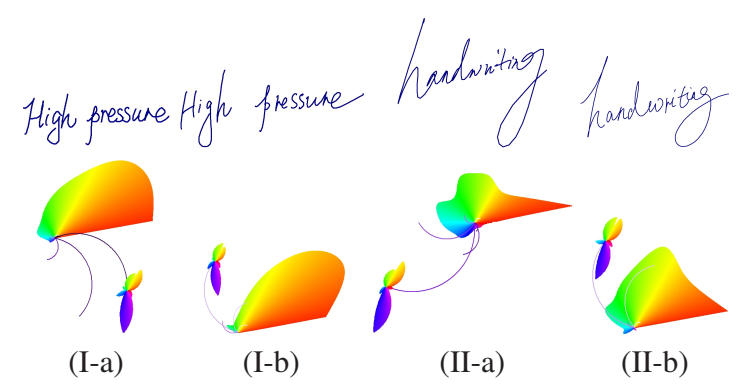

Figure 6: Two pairs of digital handwritings and their corresponding digital lilies. (I-a) is written with higher pressure than (I-b) and (II-a) is written in a faster speed than (II-b).

We first show some experimental results demonstrating that our visualization method can help bring out and reveal the characteristics of writers' individual signaturing habits. Fig. 5 shows an example where deliberate effort is made to facsimile the original handwriting, as in a forgery case. Despite the fact that the original and the facsimiled handwriting patterns are highly similar, their digital lilies look vastly different. Fig. 6 gives some comparison results which show that for similar digital signatures, their digital lilies could differ significantly as long as there is some difference in either the writing speed or the writing pressure. Specifically, even though the petals of Fig. 6(I-a) and (I-b), are quite similar, we can still easily distinguish them by observing their pistil shapes and anther locations. Thus, minute differences in the original shapes of the signature are amplified and augmented in our digital lily visualization. This facilitates signature authentication with much less user identification effort.

Now we show an experiment to demonstrate that the same person's signature will remain highly stable while different people's signatures will differ drastically. We asked forty individuals to sign their names and also to facsimile others' signatures. Fig. 7 shows some selected experiment results. Three groups of experiment results are reported here includ- ing both the handwriting pieces and their digital lilies. (I$\left.O_{1}\right)-\left(\mathrm{I}-O_{6}\right)$ are six authentic digital signatures by a person collected at six different times in a week. (I- $\left.F_{1}\right)-\left(\mathrm{I}-F_{6}\right)$ are some carefully created facsimileing results by other people in the subject group. Notice how similar and stable the signatures by the same person are through our visualization method, and how those facsimiled signatures vary in the visualization. Even though the facsimiled handwritings look very similar to the authentic ones, our visualization very clearly reveals that they are faked. In this figure, we also show the results of two more sets of similar experiments where two other individuals' digital signatures are facsimiled by other subjects. The shapes of the facsimiled signatures appear highly similar to those of the authentic ones while our digital lilies clearly tell the fake ones from the real ones.

\section{User Study}

To more objectively evaluate the effectiveness of our method, we carried out the following user study.

\subsection{Collecting Authentic Signatures and Faked Ones}

We invited 40 students (18 females and 22 males) to form our user study subject group. We built a digital signature and its corresponding digital lily collection with the help of this user group. Every time when a participant used our system to create a handwriting sample, we also asked him/her to imitate a randomly selected existing handwriting sample in our collection. This way we also gathered a collection of forged handwritings. Visualization result for each signature was immediately generated after the handwriting process and was also stored in our collection. Once these data were collected, we used them in a survey to explore the effectiveness of our method.

\subsection{Online Questionnaire}

We used an online questionnaire to evaluate the effectiveness of our visualization results, which consists of the following questions.

1. Given eight signatures, please select all the handwritings which you think are written by the person who created the first signature.

2. Given eight signatures and their corresponding digital lilies, please select all the handwritings which you think are written by the person who created the first signature.

3. Given eight pieces of handwritings, we know in advance there is one and only one person who wrote more than one piece. Please select all the handwritings which you think are written by that person.

4. Given eight pieces of handwriting and their corresponding digital lilies. We know in advance there is one and only one person who wrote more than one piece. Please select all the handwritings which you think are written by that person. 
Table 1: Question answering accuracy for $Q 1$ and $Q 2$ where $Q 1$ studies the signature authentication accuracy without using our visualization method and $Q 2$ studies the signature authentication accuracy with our visualization. Here we report the subjects' performance over six groups of questions as well as the overall performance on all the ten groups of questions.

\begin{tabular}{|c|c|c|c|c|c|c|c|c|c|c|c|c|c|c|}
\hline & Group1 & Group2 & \multicolumn{2}{c|}{ Group3 } & \multicolumn{2}{c|}{ Group4 } & \multicolumn{2}{c|}{ Group5 } & \multicolumn{2}{c|}{ Group6 } & \multicolumn{2}{c|}{ Total } \\
\hline & Q1 & Q2 & Q1 & Q2 & Q1 & Q2 & Q1 & Q2 & Q1 & Q2 & Q1 & Q2 & Q1 & Q2 \\
\hline Min & $0.0 \%$ & $85.0 \%$ & $0.0 \%$ & $75.0 \%$ & $0.0 \%$ & $86.4 \%$ & $0.0 \%$ & 79.8 & $0.0 \%$ & $84.3 \%$ & $0.0 \%$ & $76.6 \%$ & $0.0 \%$ & $81.4 \%$ \\
\hline Lower Quartile & $2.7 \%$ & $97.4 \%$ & $0.4 \%$ & $97.1 \%$ & $2.4 \%$ & $94.4 \%$ & $5.6 \%$ & $94.5 \%$ & $3.4 \%$ & $96.5 \%$ & $4.7 \%$ & $95.4 \%$ & $8.4 \%$ & $96.7 \%$ \\
\hline Median & $10.1 \%$ & $98.5 \%$ & $14.5 \%$ & $98.2 \%$ & $11.2 \%$ & $98.4 \%$ & $12.1 \%$ & $96.5 \%$ & $13.2 \%$ & $97.4 \%$ & $11.9 \%$ & $97.3 \%$ & $12.4 \%$ & $98.4 \%$ \\
\hline Higher Quartile & $25.5 \%$ & $99.6 \%$ & $31.8 \%$ & $99.9 \%$ & $24.7 \%$ & $98.7 \%$ & $24.4 \%$ & $99.5 \%$ & $19.9 \%$ & $99.2 \%$ & $23.8 \%$ & $98.7 \%$ & $32.4 \%$ & $99.8 \%$ \\
\hline Max & $51.3 \%$ & $100.0 \%$ & $54.9 \%$ & $100.0 \%$ & $50.0 \%$ & $100.0 \%$ & $54.2 \%$ & $100.0 \%$ & $44.3 \%$ & $100.0 \%$ & $54.1 \%$ & $100.0 \%$ & $52.3 \%$ & $100.0 \%$ \\
\hline Average & $14.8 \%$ & $97.9 \%$ & $23.9 \%$ & $96.9 \%$ & $16.2 \%$ & $97.6 \%$ & $23.8 \%$ & $98.1 \%$ & $26.7 \%$ & $97.5 \%$ & $10.6 \%$ & $98.2 \%$ & $\mathbf{2 0 . 4 \%}$ & $\mathbf{9 7 . 7 \%}$ \\
\hline
\end{tabular}

Table 2: Question answering accuracy for $Q 3$ and $Q 4$ where $Q 3$ explores writer verification accuracy without the assistance of our visualization method and Q4 studies the writer verification accuracy with the assistance of our visualization method. We report here all the subjects' performance over the first six groups of questions and their overall performance on all the ten groups of questions.

\begin{tabular}{|c|c|c|c|c|c|c|c|c|c|c|c|c|c|c|}
\hline & \multicolumn{2}{|c|}{ Group1 } & \multicolumn{2}{|c|}{ Group2 } & \multicolumn{2}{|c|}{ Group3 } & \multicolumn{2}{|c|}{ Group4 } & \multicolumn{2}{|c|}{ Group5 } & \multicolumn{2}{|c|}{ Group6 } & \multicolumn{2}{|c|}{ Total } \\
\hline & Q3 & Q4 & Q3 & Q4 & Q3 & Q4 & Q3 & $\mathrm{Q} 4$ & Q3 & $\mathrm{Q} 4$ & Q3 & Q4 & Q3 & Q4 \\
\hline Min & $0.0 \%$ & $86.0 \%$ & $0.0 \%$ & $85.8 \%$ & $0.0 \%$ & $83.2 \%$ & $0.0 \%$ & 74.9 & $0.0 \%$ & $84.6 \%$ & $0.0 \%$ & $72.6 \%$ & $0.0 \%$ & $82.8 \%$ \\
\hline Lower Quartile & $5.6 \%$ & $96.8 \%$ & $7.6 \%$ & $97.3 \%$ & $2.6 \%$ & $95.1 \%$ & $3.7 \%$ & $93.5 \%$ & $4.4 \%$ & $95.5 \%$ & $10.7 \%$ & $96.1 \%$ & $6.4 \%$ & $97.2 \%$ \\
\hline Median & $10.6 \%$ & $98.1 \%$ & $13.8 \%$ & $98.5 \%$ & $5.3 \%$ & $98.4 \%$ & $6.1 \%$ & $98.5 \%$ & $13.6 \%$ & $97.5 \%$ & $15.6 \%$ & $97.4 \%$ & $11.4 \%$ & $97.9 \%$ \\
\hline Higher Quartile & $26.3 \%$ & $99.7 \%$ & $32.6 \%$ & $99.6 \%$ & $16.8 \%$ & $98.7 \%$ & $28.2 \%$ & $99.3 \%$ & $21.3 \%$ & $98.2 \%$ & $22.7 \%$ & $98.7 \%$ & $24.6 \%$ & $99.5 \%$ \\
\hline Max & $42.3 \%$ & $100.0 \%$ & $46.7 \%$ & $100.0 \%$ & $50.0 \%$ & $100.0 \%$ & $54.6 \%$ & $100.0 \%$ & $42.7 \%$ & $100.0 \%$ & $56.3 \%$ & $100.0 \%$ & $42.3 \%$ & $100.0 \%$ \\
\hline Average & $15.7 \%$ & $97.1 \%$ & $15.2 \%$ & $98.3 \%$ & $14.1 \%$ & $97.7 \%$ & $10.8 \%$ & $96.5 \%$ & $13.8 \%$ & $96.8 \%$ & $18.7 \%$ & $98.2 \%$ & $12.4 \%$ & $97.3 \%$ \\
\hline
\end{tabular}

5. What is your age?

6. What is your major?

7. Are you familiar with digital handwriting or digital signature before participating in this study? [0-5]: 0 , Never encountered; 5, Yes, very familiar.

8. Do you feel our digital lily is easy to perceive? [0-5]: 0 , No, too overwhelming; 5, Yes, very clear.

9. How do you feel about the visual appearance of our visualization? Are you interested in looking at them? [0-5]: 0 , Not interested at all; 5, Highly interested.

10. Do you wish to apply our current visualization method to assist handwriting authentication in real life? [0-5]: 0, Strongly object; 5, Strongly support.

In the above, Questions 1 to 2 evaluate the effectiveness of our visualization method for signature authentication. Questions 3 to 4 evaluate the effectiveness of our visualization method for handwriting writer identification. Both pairs of questions intend to show the effectiveness of our method through the improvement of the correct human recognition rate after employing our visualization method, as opposed to relying on only the digital signatures. Questions 5-7 survey the user's background. Question 8 studies whether the user feels our visualization method is easy to use. Question 9 is on the aesthetics and visual attraction of our visualization results. Question 10 surveys people's judgement on the maturity and readiness of our technology for practical deployment. The above questions can be broadly classified into two parts where Questions 1-4 form the first part and Questions 5-10 form the second part. To generate a questionnaire, questions in the second part will only occur once while questions in the first part can be repeated for multiple times; of course each time the digital handwriting and its digital lilies will be changed.

\subsection{User Study Results}

We invited another 100 individuals to participate in our online user survey using the questionnaire shown above. These individuals were newly invited and none of them were in our signature acquisition group (see Sec. 5.1). We randomly selected 10 groups of handwriting pieces and their corresponding digital lilies to form Questions 1-4. In each group, there are eight handwriting pieces where $x$ pieces are the authentic handwriting pieces by the same person and $(8-x)$ are forged handwriting pieces by other people whom were also randomly selected from our collection. Here $x$ is a random integer between 2 and 6 . In this way, each time when a participant accessed our online questionnaire, the survey server will randomly select a group of digital handwritings and their digital lilies to dynamically generate the survey webpage. After the participant answered a question, his/her result will be automatically saved and evaluated on our server.

Due to space limit, we report here the accuracy achieved by 6 out of 10 survey groups along with the overall accu- 
Table 3: Performance comparison between the accuracies of automatic signature authentication methods as reported in the literature and the accuracy of our method.

\begin{tabular}{|c|c|c|c|c|}
\hline [KA01] & [SVD04] & [SB07] & [BNPH02] & Our Method \\
\hline $93.2 \%$ & $96.0 \%$ & $96.56 \%$ & $95.3 \%$ & $\mathbf{9 7 . 7 \%}$ \\
\hline
\end{tabular}

racy achieved by all the 10 groups. Table 1 reports the answering accuracy for Questions 1 and 2. Table 2 reports the answering accuracy for Questions 3 and 4 . According to the data reported in Table 1, we can see that without the help of our visualization method, the average accuracy of signature authentication as measured by Question 1 is 20.4\%, while the average accuracy of signature authentication with the help of our visualization method as measured by Question 2 is $97.7 \%$. This result is better than the result reported in [SB07], where the recognition accuracy is $96.56 \%$. For Questions 3 and 4 whose results are reported at Table 2, without the assistance of our visualization method, the average accuracy as measured by Question 3 is $12.4 \%$, while with our visualization assistance, the average accuracy as measured by Question 4 is $97.3 \%$. In addition, we also compare the performance of other automatic signature identification algorithms to the average performance of our method, whose results are reported in Table 3. These data confirm the superiority of our method with respect to the state-of-art automatic signature authentication algorithms. Note that according to Table 1, the question answering quality for Question 2, i.e., the signature authentication accuracy with the assistance of our visualization method, has an average of $97.7 \%$, a lower quartile value of $96.7 \%$ and a higher quartile value of $99.8 \%$. Recall this is the performance of all the 100 subjects participating in our study. We can therefore arrive at a statistically robust conclusion that our method outperforms these existing automatic signature authentication methods in the literature. Similarly, according to Table 2, the majority of the writer verification accuracy with the assistance of our visualization method is above $97.2 \%$ (the lower quartile value), which is superior to existing automatic methods.

Lastly, from the statistics of Questions 7-10 as shown in Table 4 , we can see that $81 \%$ of the participants think our visualization is easy to perceive and use; $73 \%(=46 \%+27 \%)$ of the participants are interested in using our visualization method to help them authenticate signatures; and $51 \%$ of them want to see our visualization results being applied in reality for signature authentication. This indicates a high acceptance of our visualization method by the user group.

\section{Conclusion}

In this paper, we introduce a new metaphor called "digital lily" for visualizing human handwriting characteristics in a digital signature process. In designing our visualization method, we consider both the expressiveness and the
Table 4: Statistics of answers to Questions 5, 7-10.

\begin{tabular}{|c|c|c|c|c|}
\hline Q5 & $15-20: 22 \%$ & $21-25: 42 \%$ & $26-30: 24 \%$ & $>30: 12 \%$ \\
\hline \multirow{2}{*}{ Q7 } & $\begin{array}{c}\text { Never encou- } \\
\text { ntered: } 26 \%\end{array}$ & $\begin{array}{c}\text { Only heard } \\
\text { about it: 38\% }\end{array}$ & $\begin{array}{c}\text { Seldomly } \\
\text { used: } 24 \%\end{array}$ & $\begin{array}{c}\text { Familiar: } \\
12 \%\end{array}$ \\
\hline \multirow{2}{*}{ Q8 } & $\begin{array}{c}\text { Too overwh- } \\
\text { elming: 3\% }\end{array}$ & $\begin{array}{c}\text { Difficult: } \\
6 \%\end{array}$ & $\begin{array}{c}\text { Neutral: } \\
10 \%\end{array}$ & $\begin{array}{c}\text { Clear: } \\
\mathbf{8 1 \%}\end{array}$ \\
\hline \multirow{2}{*}{ Q9 } & $\begin{array}{c}\text { Uninterested: } \\
12 \%\end{array}$ & $\begin{array}{c}\text { Neural: } \\
15 \%\end{array}$ & $\begin{array}{c}\text { Interested: } \\
\mathbf{4 6 \%}\end{array}$ & $\begin{array}{l}\text { Highly inte- } \\
\text { rested: 27\% }\end{array}$ \\
\hline \multirow{2}{*}{ Q10 } & Reject: & $\begin{array}{c}\text { Probably } \\
\text { reject:12\% }\end{array}$ & $\begin{array}{c}\text { Neural: } \\
29 \%\end{array}$ & $\begin{array}{c}\text { Accept: } \\
\mathbf{5 1 \%}\end{array}$ \\
\hline
\end{tabular}

aesthetics of the metaphor. According to our experiment results, we find that the visual pattern generated using our method is persistently stable for signatures created by the same person; whereas for signatures facsimiled by others, even though their shapes are highly similar, the generated digital lilies reveal clearly that they are different. Through a comprehensive user study we further confirm that our visualization method can effectively help people more intuitively and reliably authenticate digital signatures. Our work therefore suggests that visualization can usefully enhance the security of digital signatures, which is a topic worthy of more future work.

\section{Acknowledgement}

We thank Li Yuen Kwan for helping us recruit subjects to participate in our user studies. This work has a patent pending.

\section{References}

[BNPH02] Bensefia A., Nosary A., Paquet T., Heutte L.: Writer identification by writer's invariants. In Proc. of Frontiers in Handwriting Recognition (2002), pp. 274-279.

[BPH05] Bensefia A., Paquet T., Heutte L.: A writer identification and verification system. Pattern Recognition Letters 26, 13 (2005), 2080-2092.

[BS07] Bulacu M., SCHOMAKer L.: Text-independent writer identification and verification using textural and allographic features. IEEE Trans. Pattern Anal. Mach. Intell. 29, 4 (2007), 701717.

[KA01] Kikuchi M., Akamatsu N.: Development of speedy and high-sensitivity pen system for writing pressure and writer identification. Systems and Computers in Japan 32, 6 (2001), 1763-1772.

[SB04] SchomaKer L., Bulacu M.: Automatic writer identification using connected-component contours and edge-based features of uppercase western script. IEEE Trans. Pattern Anal. Mach. Intell. 26, 6 (2004), 787-798.

[SB07] SchlapBACH A., BUNKe H.: A writer identification and verification system using hmm based recognizers. Pattern Analysis and Applications 10, 1 (2007), 33-43.

[SVD04] Schimke S., Vielhauer C., Dittmann J.: Using adapted levenshtein distance for on-line signature authentication. In Proc. of International Conference on Pattern Recognition (2004), pp. 931-934. 
Songhua Xu, Wenxia Yang, and Francis C.M. Lau / A Visualization Based Approach for Digital Signature Authentication

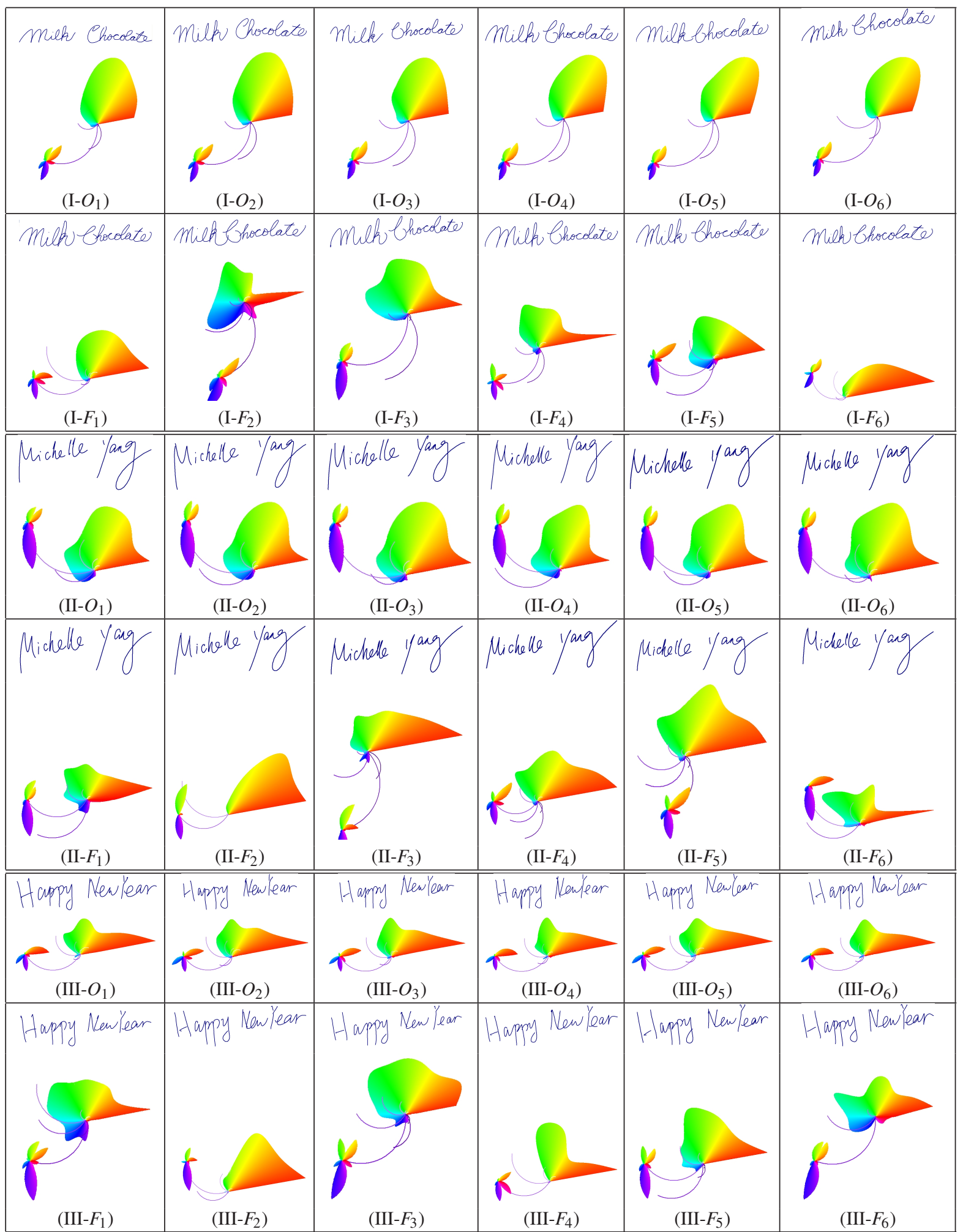

Figure 7: Three groups of authentic digital signatures and their facsimiles. $(O)$ stands for the authentic signatures by the original person and $(F)$ stands for facsimiled results by others. 